\title{
Evaluation of near patient testing for Chlamydia trachomatis in a pregnancy termination service
}

\author{
Jennifer Hopwood, MB ChB, D(Obst)RCOG, Dip Ven, MFFP, Senior Clinical Medical Officer, Wirral and West Cheshire Community \\ NHS Trust, Co-ordinator Wirral DH Chlamydia Pilot Scheme, St Catherine's Hospital, Wirral, UK; Harry Mallinson, PhD, \\ Principal Microbiologist, University Hospital, Aintree, UK; Toni Gleave, RGN BA (Hons) MSc, Community Health Adviser, St \\ Catherine's Hospital, Wirral, UK.
}

Correspondence: Dr JJ Hopwood, Chlamydia Pilot Office, Evidence Based Practice Centre, St Catherine's Hospital, Birkenhead, CH44 OLQ.

(Accepted $2^{\text {nd }}$ February 2001)

The Journal of Family Planning and Reproductive Health Care 2001: 27(3): 127-130

\begin{abstract}
Aim. To identify and evaluate advantages and disadvantages of a near patient test (NPT) for Chlamydia trachomatis, using Clearview Chlamydia MF (Unipath Ltd) in a British Pregnancy Advisory Service (BPAS) clinic.

Method. The improved Clearview Chlamydia MF test was used to test endocervical swabs from 400 women attending $B P A S$ clinic for termination of pregnancy. The results were compared with Ligase Chain Reaction (LCR), using Polymerase Chain Reaction (PCR) as the arbiter.

Results. Twenty-seven women tested positive by Clearview Chlamydia MF (24 confirmed by LCR) and 32 by LCR.

Comment. NPT has potential advantages in specific situations where a quick result is required for optimal management of those testing positive. However, the current technology available for detection of Chlamydial infection results in time constraints, which limited its benefits in this study, where there was a high throughput of clients.

A significant number of cases were missed by Clearview Chlamydia MF, though the sensitivity found is within the ranges reported for various enzyme immunoassays (EIA) currently the most commonly used testing method.
\end{abstract}

The study confirmed the high positivity in those attending for termination, especially in under 25-year-olds.

\section{Key message points}

- A termination service provides an effective health care opportunity for finding chlamydial infection.

- This study confirms that the prevalence of Chlamydia trachomatis in over 25-year-olds is significantly less than in younger women.

- Twenty-six percent of women would have been especially likely to benefit from near patient testing.

- Clearview chlamydia MF results were available to be given before discharge from the clinic in $76 \%$ of cases.

- If a designated person can be used, the Clearview chlamydia technique can be incorporated effectively into a service, but there needs to be adequate time allocated to gain maximum benefit.

\section{Introduction}

Chlamydial infection is the most common bacterial sexually transmitted infection (STI) and the most common cause of pelvic inflammatory disease (PID). Estimates of the incidence of PID after termination of pregnancy in infected women range from $25 \%$ to $63 \%$. ${ }^{1}$ BPAS is a major national specialist provider of abortion services, offering diagnosis, information and counselling to women about being pregnant and, where requested and appropriate, treatment for termination of pregnancy. Founded after the Abortion Act of 1967, the charity now provides counselling and clinical assessment from a network of BPAS centres and conducts treatment in 12 Department of Health approved clinics. The interval between assessment and treatment may be only a few days for some clients.

Recognising the risk of ascending infection associated with instrumentation, it has always been the policy of BPAS to give prophylactic antibiotics, currently a course of doxycycline. This policy does not address the problem of partners, so for those with the infection re-infection is likely, and from the public health aspect, the prevalence of C. trachomatis is not likely to be effectively reduced in the community. There is a view, therefore, that testing for Chlamydial infection should be offered prior to termination so that appropriate advice can be offered when a test is positive. This is supported by the Chief Medical Officers report $^{2}$ which recommends that 'screening should be offered to all women seeking termination of pregnancy and their partners. Screening is especially important for termination of pregnancy clients as the procedure increases the risk of pelvic inflammatory infection in women who are infected with $C$. trachomatis

The performance of a test is often described in the terms of its sensitivity and specificity. These depend not only on the intrinsic qualities of the test, but also on the sample supplied, storage and transport conditions. The test must be acceptable and affordable and the results should be available so that appropriate and timely information and management can be given. In our previous study with 587 women $^{3}$ using urine tested by Ligase Chain Reaction (LCR) at pregnancy counselling, together with endocervical swabs at the time of termination, we confirmed that after leaving the clinic, many were difficult to contact with their results. Women are referred and self-refer from a wide geographical area. They may not give their true address or want to be contacted after leaving the service.

Near patient testing (NPT) is any investigation carried out in a clinical setting or patient's home for which the result is available without reference to a laboratory and perhaps rapidly enough to affect immediate patient management. ${ }^{4}$

Quality control is as important in NPT as it is in a laboratory. Important issues to address include inter- and intra-observer variability and avoidance of cross contamination. 
Clearview chlamydia $\mathrm{MF}$ is a rapid monoclonal antibody-based latex visual end point test, which works using immuno-chromatographic technology and is similar in appearance to the Clearview HCG II pregnancy test (Figure 1). The latter is fairly simple in that urine is a relatively uncontaminated sample and the test is for one relatively simple component, human chorionic gonadotrophin. Testing for C. trachomatis, however, is a technical challenge, as it is a complex organism from which lipopolysaccharide (LPS) antigen must be extracted by heating the sample. A further problem in non-amplification tests is that a swab sample is required which contains many contaminants. Once LPS has been extracted, the Clearview chlamydia MF test is then a one step process (some other manufacturers systems require additional reagents.)

The test in the past has not been regarded as sufficiently sensitive. ${ }^{5}$ Sensitivity and specificity have previously been measured/calculated using culture as the comparator and FDA approval rests on this comparison.

There is a new extraction procedure which reduces the interference caused by soluble components in the sample, but which still produces a reactive form of the chlamydial LPS. The undoubted attraction of a rapid result led us to examine the performance of this improved technology and to compare it against LCR, which has greater sensitivity than culture in most clinical situations.

\section{Figure 1 Clearview Chlamydia MF (Unipath)}

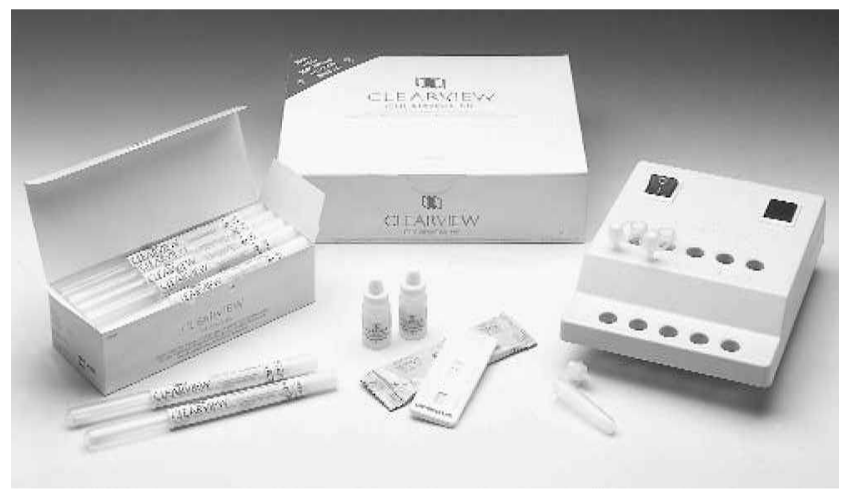

\section{Method}

Ethics Committee approval for the study was obtained.

During February and March 2000, all women attending for surgical termination of pregnancy at the Merseyside BPAS clinic, were given information about chlamydial infection verbally and on posters and information leaflets. They were also given the option of participating in the study. Modified request forms were designed and used to collect demographic and clinical information, including post-code, chosen method of obtaining the result, and gestation of the pregnancy. These each carried a sticker showing in equal numbers, which swab should be taken first.

Three surgeons, all with previous training and experience of taking such samples, collected two endocervical swabs from each woman, in random order, immediately before the termination procedure. The swabs for Clearview chlamydia MF were taken to the on-site mini laboratory and tested in batches by the biomedical scientist, who was on duty to carry out routine blood tests. The LPS extraction unit holds up to five specimens at a time. After heating to extract the antigen, the samples were tested according to manufacturer's instructions.
Results were recorded as 'positive', 'negative', 'weakly positive' when the line was faint, and 'indeterminate' when there was no line but a 'hint' of colour. Other information recorded was batch size, the time taken to test, and when the result was available.

The other swabs were held at $4{ }^{\circ} \mathrm{C}$ and transported daily to the Liverpool Public Health Laboratory (PHL) to be tested by LCR (Abbott Laboratories LCx system). Positive results were confirmed by repeat LCR from the same sample. When a result was obtained which was different from the Clearview result, Polymerase Chain Reaction (PCR) (Roche COBAS) testing was carried out at Manchester PHLS.

In this study, results were given only after the LCR result was available. A positive Clearview and/ or LCR result was given as positive. Women agreeing to have the test were asked whether they would prefer their results to be sent to their home or another address, or to be given by telephone This was recorded on the laboratory request form. For those testing positive, and where correspondence had been agreed, a letter and information leaflet about chlamydial infection for patient and partner, together with opening times of their local Department of Genitourinary Medicine, were sent by post. All women having a termination were given routine antibiotic prophylaxis in the clinic as usual.

The case load of the service was obtained from computer records.

\section{Results}

In the time period, 400 women were tested, 399 by LCR and Clearview (one woman did not have an LCR swab taken) (Table 1).

Table 1 Results of Clearview Chlamydia MF and LCR tests

\begin{tabular}{lllllll}
\hline \multicolumn{7}{c}{ Clearview Chlamydia MF } \\
& Negative & Positive & Weak Pos. & Indeterminate & Total \\
\hline \multirow{4}{*}{ LCR } & Negative & 360 & 0 & $3 * *$ & $4 * * *$ & 367 \\
& $\begin{array}{l}\text { Positive } \\
\text { Total }\end{array}$ & $8^{*}$ & 16 & 8 & 0 & 32 \\
\hline
\end{tabular}

* 7 confirmed by PCR

** PCR negative

*** PCR inhibitory

Three hundred and sixty tests were negative by both Clearview and LCR and 24 were positive by both (for eight of these Clearview was weakly positive).

Of the 11 reported as 'weakly positive' by Clearview, three were negative by LCR and PCR, so the women were possibly overcalled. Four tests were reported as 'indeterminate' by Clearview, these were negative by LCR and did not give a valid PCR result due to the presence of inhibitors, indicated by control tests carried out on each sample.

Of the eight tests that were Clearview negative/LCR positive, one was not confirmed by PCR. On re-testing by LCR this one was found to be at the cut-off for a positive reading.

Clearview missed eight of 32 (26\%) LCR positive cases i.e. found 16 definitely and eight weakly.

There is a high prevalence of chlamydial infection in women presenting for termination of pregnancy, reflecting that they represent a group who have by definition recently been sexually active, but not necessarily that they are leading more risky lifestyles. More information would be 
required for better interpretation of this common finding. This study confirms that the prevalence of chlamydial infection in over 25 -year-olds is significantly less than in the younger women (Table 2).

Table 2 Clearview Chlamydia MF and LCR positive results by age band

\begin{tabular}{lcccccc}
\hline & $\begin{array}{c}15-19 \\
\text { years }\end{array}$ & $\begin{array}{c}20-24 \\
\text { years }\end{array}$ & $\begin{array}{c}25-29 \\
\text { years }\end{array}$ & $\begin{array}{c}30-34 \\
\text { years }\end{array}$ & $\begin{array}{c}35-39 \\
\text { years }\end{array}$ & $\begin{array}{c}40-44 \\
\text { years }\end{array}$ \\
\hline Clearview & $9 / 89$ & $11 / 119$ & $4 / 65$ & $1 / 67$ & $0 / 29$ & $0 / 9$ \\
& $(10.1 \%)$ & $(9.2 \%)$ & $(6.2 \%)$ & $(1.5 \%)$ & & \\
LCR & $10 / 89$ & $16 / 119$ & $4 / 65$ & $0 / 67$ & $1 / 29$ & $0 / 9$ \\
& $(11.2 \%)$ & $(13.4 \%)$ & $(6.2 \%)$ & & & \\
\hline
\end{tabular}

\section{Observations on Clearview testing}

Clarity of reading. Of the Clearview tests, 11 were reported as 'weakly positive', eight of which were LCR positive. The three that were LCR negative were also PCR negative. Only four $(1 \%)$ were reported as 'indeterminate', and these all tested negative by LCR. Interestingly, these samples gave invalid results by PCR due to the presence of inhibitors, indicated by control tests carried out on each sample. For any test, the percentage of samples requiring a repeat test to clarify or confirm is an important factor in evaluating its performance.

Time to test. The time was measured from placing the samples in the heated workstation until reading the results. Processing was completed in 40 minutes or less for $45 \%$ of batches, and $92 \%$ were processed in less than an hour. Two thirds of batches were processed between 36 and 50 minutes.

Service implications of the testing process. The time from admission of a client to her discharge from the service is approximately 3.5 hours, of which only $1-1.5$ hours is postoperation i.e. post-sampling time.

Priorities of care meant that tests could not, logistically, have been carried out on an individual basis by the nurse assigned to the client, and so the biomedical scientist on duty at the clinic was the person best placed to do the testing. The samples were taken from theatre to the on-site mini laboratory. It was not feasible to test each sample individually, so it was necessary to wait until four or five tests accumulated. Transferring the samples from theatre and waiting for batch testing built in a delay. Most samples $(83.5 \%)$ were processed in a batch size of five samples and only two tests were carried out singly. The samples could not, therefore, all be tested before the end of the scientists' working day. Ninety-six tests $(24 \%)$ were not able to be carried out on the day of the operation so the results would not have been available before discharge from the clinic.

An important factor in the logistics of this work was that during March 2000 there was a $25 \%$ increase in numbers of terminations carried out in this BPAS clinic with the accompanying increased routine workload for the scientist. Clearview testing was found not to be a desktop procedure in this situation. It was possible to incorporate it into the service, but it would require extra time or personnel to realise its potential.

Transmitting results. NPT should allow results to be given immediately. Laboratory based tests require a mechanism for transmitting results later.
In this study, $270(68 \%)$ of the women tested asked for their result to be sent to their home address, and $23(5.8 \%)$ to another address. The $104(26 \%)$ of women who would have been harder to reach, and therefore especially likely to benefit from NPT, comprised 15 (3.8\%) who gave a contact telephone number, $80(20.2 \%)$ who wanted to phone the clinic, and nine $(2.3 \%)$ who wanted to be contacted at various other places.

\section{Discussion}

A termination service provides an effective health care opportunity for finding Chlamydial infection, in addition to its special responsibility in minimising the effect of instrumentation of the uterus. So, as an adjunct to antibiotic prophylaxis, testing should be considered in order to identify women with the infection and to enable treatment to be given to their partners to avoid re-infection.

It is necessary in evaluating a test to examine all aspects of its performance. These include the resources and time to carry out the test, and the method of transmitting the results. If the results are available before discharge from the service, time is required to give them and to counsel the client appropriately. If the results are not available before discharge, then time is needed to trace clients and to discuss results with those who telephone, and there is the certainty that some will never access their results.

Clearview Chlamydia MF results were available to be given before discharge from the clinic in $76 \%$ of cases. In this study, the technique was incorporated effectively into the service by having a designated person to carry out the tests, but there does need to be adequate time allowed to gain maximum benefit. Clearview missed eight $(26 \%)$ of 32 LCR positive cases i.e. found 16 plus eight weakly, though possibly by overcalling three, as three were negative by LCR and PCR. Only $1 \%$ gave indeterminate readings. Although cases were missed, its sensitivity lies within the ranges $(55 \%-85 \%)$ reported for various enzyme immunoassays (EIA) ${ }^{6,7}$ - currently the most commonly used testing method.

The control of chlamydial infection would be revolutionised by a simple NPT so further improvements in technology will be welcomed.

\section{Acknowledgements}

Paulette Green, Biomedical Scientist who carried out all the Clearview tests. Kally Worthington, Manager Merseyside BPAS and Rebecca Wood, Liverpool PHLS for their work on the project. Lesley Foster, Unipath and Ian Jones, Chief Executive BPAS for their help and support. Ian Simms, PHLS CDSC for his comments.

\footnotetext{
Statements on funding and competing interests

Funding. Clearview testing kits and laboratory LCR tests wre funded by Unipath.

Competing interests. None.

References

Moller BR, Ahrons S, Laurin J, et al. Pelvic infection after elective abortion associated with Chlamydia trachomatis. Obstet Gynaecol 1982; 59: 210-213.

Chief Medical Officer's Expert Advisory Group. Main report of the CMO's expert advisor group on Chlamydia trachomatis. London: Department of Health, 1998.

3 Hopwood J, Mallinson M, Jones I. There is more to a test than technology - evaluation of testing for chlamydia infection in a charitable sector termination service. Br J of Fam Plann 1998; 23 $116-119$.

4 Best LM, Veldhuyzen van Zanten SJ, Sherman PM, et al. Serological detection of Helicobacter pylori antibodies in children and their parents. J Clin Microbiol 1994; 32:1193-196.

5 Thomas BJ, Macleod EJ, Taylor-Robinson D. Evaluation of Sensitivity of 10 diagnostic assay for Chlamydia trachomatis by use of a simple laboratory procedure. J Clin Path 1993; 46: 912-914.

6 Black C. Current methods of laboratory diagnosis of Chlamydia trachomatis infections. Clinical Microbiological Reviews Jan 1997 : 160-187.

Robinson AJ, Ridgway GL. Modern diagnosis and management of genital Chlamydia trachomatis infection. British Journal of Hospital Medicine 1996; 55 (7): 388-393.
} 